\title{
Braurechte und Bierproduktion am Anfang des 16. Jahrhunderts in der Stadt Torgau \\ Eine Auswertung der Tranksteuerregister
}

\author{
von \\ JÜRGEN HERZOG
}

Das Torgauer Bier hat über Jahrhunderte das Wirtschaftsleben der Stadt maßgeblich geprägt und den Wohlstand der Bürger in besonderem Maße begründet. Begrifflich findet das in der Bezeichnung ,Bier als Brot der Stadt' seinen Niederschlag. Die stadtgeschichtliche Forschung hat sich mit diesem Thema häufig auseinandergesetzt. ${ }^{1}$ Dabei wird immer wieder auf die umfangreichen Tranksteuerregister im Thüringischen Hauptstaatsarchiv Weimar hingewiesen, ohne dass eine geschlossene Auswertung dieser Bestände bisher erfolgt ist. Den folgenden Betrachtungen liegen die Tranksteuerregister der Stadt Torgau der Jahre 1514 bis 1525 zugrunde. ${ }^{2}$ Die Register führen alle Brauberechtigten in immer wiederkehrender Reihe auf, einer Reihenfolge, die mit der jahrhundertelangen Praxis anderer Steuerregister übereinstimmt. Damit können die Brauberechtigten weitestgehend zweifelsfrei den Hausgrundstücken zugeordnet werden. ${ }^{3}$

\section{Die Tranksteuern von 1514 bis 1525}

Nachdem die Landstände auf dem Landtag zu Altenburg 1514 eine Tranksteuer zunächst für vier Jahre bewilligt hatten, wurde am 21. September 1514 im Ausschreiben Kurfürst Friedrichs des Weisen u. a. geregelt:

1 Besonders zu erwähnen sind: ERnst Henze, Torgisch Bier, in: Veröffentlichungen des Altertumsvereins zu Torgau 11 (1897); Rudolf Mielsch, Die sächsische Bierbrauerei im 16. und 17. Jahrhundert, in: Wissenschaftliche Beilagen des Dresdner Anzeigers 94/6 (1930); Agnes Bartscherer, Von der Entwicklung des Handwerks im einstigen Amt Torgau, in: Meine schöne Heimat, Kiel 1932; Hans-Joachim Kadatz, Bier und Brauwesen in Torgau (Schriften des Torgauer Geschichtsvereins 4), Torgau 2001.

2 Es handelt sich um den Bestand im Thüringischen Hauptstaatsarchiv Weimar, Ernestinisches Gesamtarchiv (im Folgenden: HStA Weimar, EGA), Reg Qq Abt. B Nr. 3913 - 3927, der im Folgenden nicht einzeln angemerkt wird. Vollständige Register liegen für die Steuerjahre 1514/15, 1515/16, 1517/18 und 1523/24, unvollständige für 1518/19, $1521 / 22,1522 / 23$ und $1524 / 25$ vor.

3 Verwendet wurde dazu Karl Heinz Lange, Häuserbuch der Stadt Torgau, auf Datenträger 2007. 
- den zehnten Pfennig von allem Getränk zu geben (Bier, Wein, Branntwein)

- von jedem Eimer ausgeschenkten und ausgetrunkenen Biers einen silbernen Zinsgroschen zu zahlen

- das Schenkmaß um den 10. Teil zu verringern, solange die Steuer gegeben wird.

Es handelte sich um eine Verbrauchssteuer, die dort zu entrichten war, wo innerhalb des Landes ausgeschenkt wurde. Nicht betroffen waren die Verkäufe an Abnehmer außerhalb des Kurfürstentums und an Einzelpersonen, die keinen Ausschank betrieben. Die produzierten Mengen an Nachbieren aus weiteren Aufgüssen, nach dem ersten Aufguss der Biererzeugung gewonnen - das sogenannte Sanftbier und Kofent, im Allgemeinen in den Torgauer Quellen als Trinken bezeichnet - wurden nicht besteuert und entziehen sich damit einer Auswertung. Mit der Steuereinnahme im lannd zu Meyssen, d. h. dem Hauptkreis Meißen, wurden der Torgauer Amtsschösser und der Rat zu Torgau neben dem Amtmann und Rat in Altenburg beauftragt. Nachdem die Landstände 1518 einer Verlängerung der Steuer zugestimmt hatten, erließen Kurfürst Friedrich und Herzog Johann ein nochmaliges Ausschreiben mit Verhütungsmaßregeln gegen das unordentliche Einbringen. 1525 wurde die Steuer mit der Maßgabe aufgehoben, eine andere Abgabe - eine hulff - erheben zu wollen. Der Torgauer Rat bedankte sich dafür bei den Landesherren und bat um eine gnedige bülff, da sie sonst keine nabrung dann mit bierbrauen haben, welche narung inen etlich jabr ber sebr umbgeschlagen. ${ }^{4}$

\section{Braurechte der Stadt}

Das Braurecht gehörte zu den ältesten und wichtigsten Vorrechten der städtischen Bürgergemeinden. Für Torgau kann davon ausgegangen werden, dass um 1200 die ursprüngliche Braurechtsbesetzung erfolgte, was spätere Veränderungen nicht ausschloss. Insofern erlauben die Braurechte Rückschlüsse auf die Entstehung und den ursprünglichen Umfang der frühen Bürgergemeinde. Dem bisherigen Forschungsstand liegt eine durch Karlheinz Blaschke 1968 angefertigte Braurechtskarte zugrunde, die auf dem erblichen Braurechtsbestand des Jahres 1835 beruht. $^{5}$

Aus den Tranksteuerregistern der Jahre 1514 bis 1525 lassen sich zwar die auf den Grundstücken, den Brauerben, liegenden erblichen Rechte nicht ermitteln, wohl aber die Anzahl der tatsächlich gebrauten Biere (Gebräude). Der Rekons-

4 Carl August Hugo Burkhardt, Ernestinische Landtagsakten (Thüringische Geschichtsquellen 8), Bd. 1, Jena 1902, S. 106 ff.; HStA Weimar, EGA, Reg Q 33, Kurfürstliches Ausschreiben 1514.

5 Karlheinz BlaschKe, Geschichtliche Einleitung. Die geschichtliche Entwicklung der Stadt Torgau von den Anfängen bis zum Beginn des 19. Jh., in: Die Denkmale der Stadt Torgau, Leipzig 1976, S. 13-37, hier S. 20. 
truktion einer Braurechtskarte bei Verwendung des heutigen Stadtplans, jedoch mit den alten Gassenbezeichnungen, wurden die jeweils höchsten jährlichen Braumengen dieser Zeit zugrunde gelegt (Abb. 1 und Anlage).

Mit späteren Angaben verglichen, ergibt sich folgendes Bild: ${ }^{6}$

\begin{tabular}{|l|l|l|}
\hline & Brauherrenanzahl & Bieranzahl \\
\hline $1514 / 25$ & 268 & 1.475 \\
\hline & Brauerben & \\
\hline 1638 & 271 & 1.528 \\
\hline 1705 & 268 & 1.463 \\
\hline 1835 & 245 & 1.354 \\
\hline
\end{tabular}

Tab. 1: Entwicklung der Anzabl der Brauerben und Gebräude.

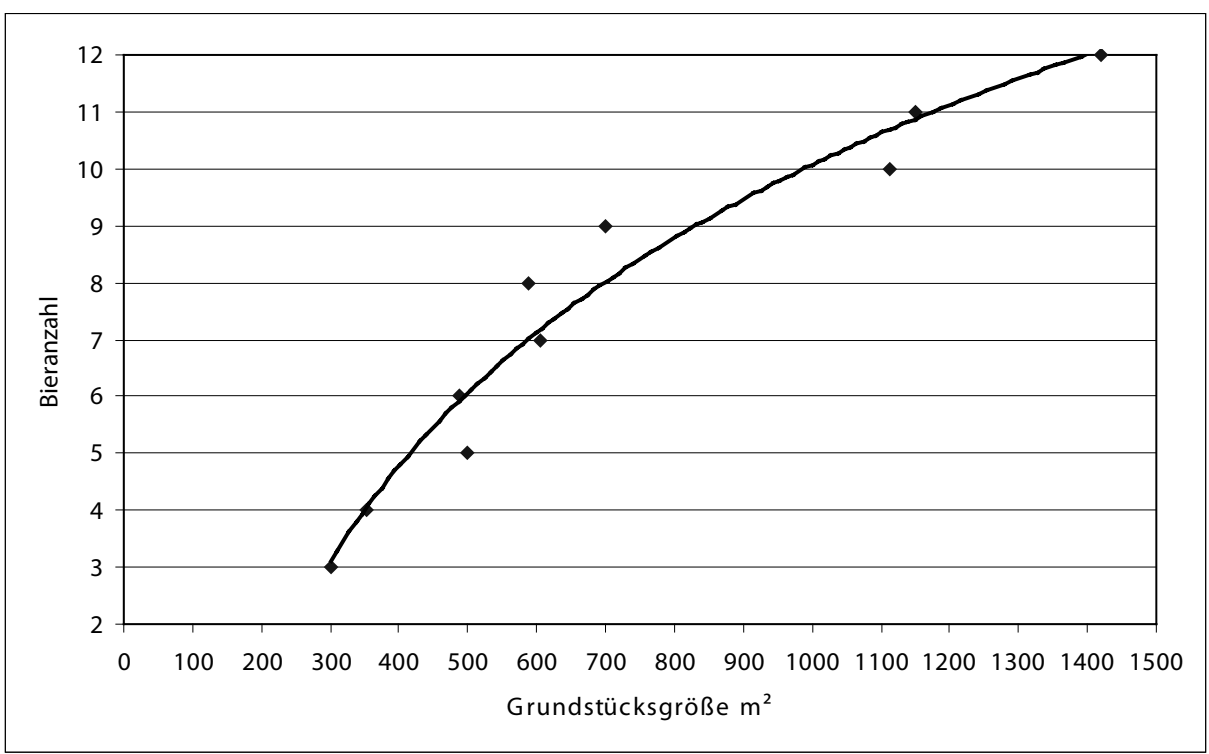

Diagramm 1: Abhängigkeit der Braurechte von der Grundstücksgröße.

6 Landeshauptarchiv Sachsen-Anhalt, Rep. D Torgau, Anh. 7 Nr. 326, Register der Kommune und steuerbaren Häuser in Torgau, 1638; Stadtarchiv Torgau, H 3294, Nachweisung der besteuerten Häuser in der Stadt, undatiert, um 1705; und B 372, Braurechte 1835. 


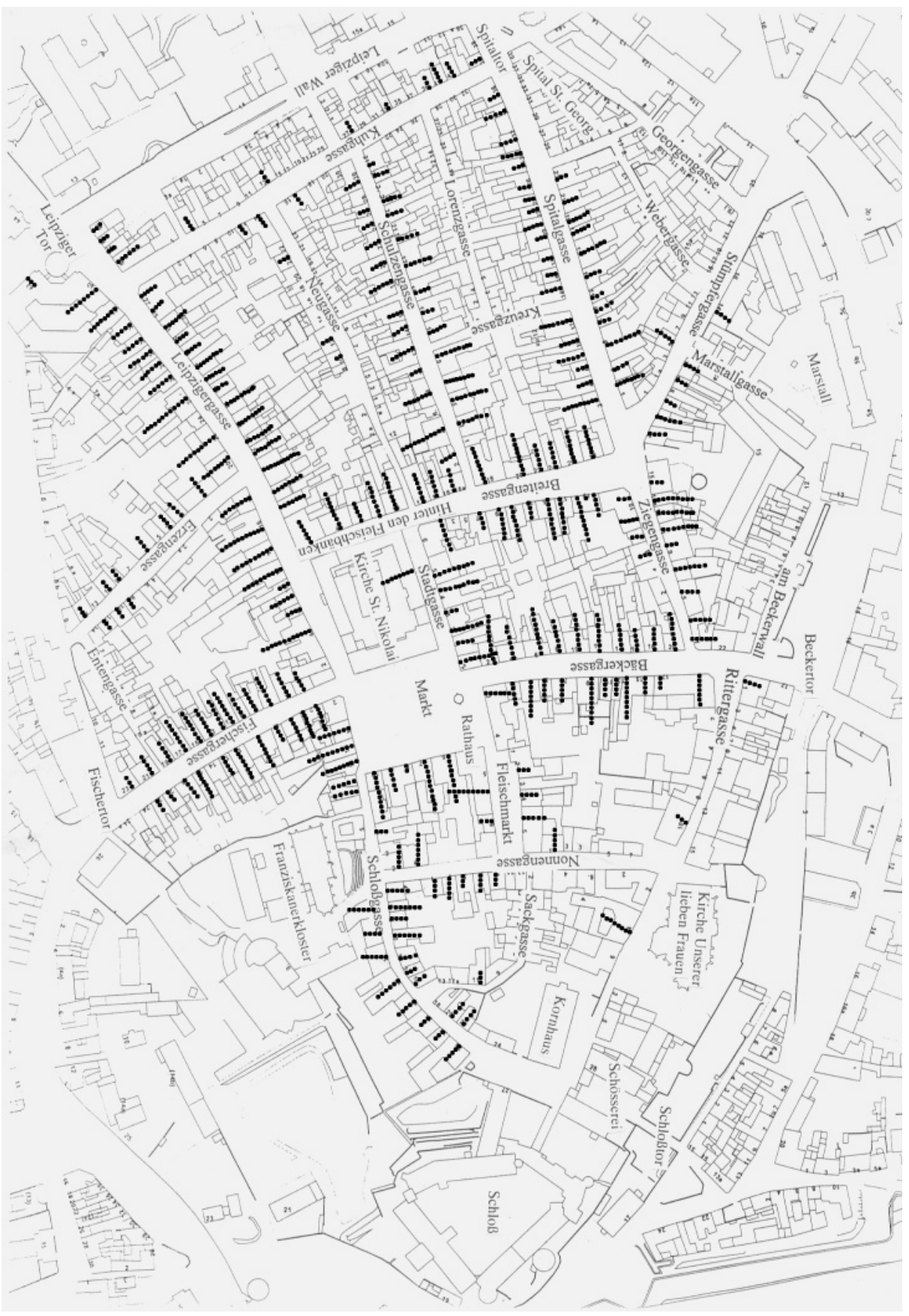

Abb. 1: Höchstanzabl der Gebräude auf den Grundstücken der Stadt 1514/25. 
$\mathrm{Zu}$ den Brauherrschaften gehörten 1514/15 der kurfürstliche Hofmeister Hans von Holdau und der Baumeister Paul von Dolenstein, die wohl kurfürstliche Gnadenbiere brauten. Die übrigen 266 waren bürgerliche Brauerben.

Bis 1628 hat ein geringer Anstieg durch zuerworbene Braurechte in Höhe von 3,5\% stattgefunden. Bis 1835 trat eine Verminderung um $8 \%$ ein, jedoch waren immer noch $73 \%$ aller grundstückbezogenen Braurechte gegenüber 1514/25 unverändert.

Eine Gegenüberstellung der ermittelten Braurechte zu den durchschnittlichen Grundstücksgrößen stützt die Annahme, dass die Höhe der Braurechte bei ihrer Vergabe von der Größe der Grundstücke abhängig war. ${ }^{7}$

Die Aufschlüsselung der Braurechte nach Gassen im Vergleich zur steuerlichen Bewertung von 1531 bestätigt die Tendenz der Grundstücksbewertung in Abhängigkeit von der Braurechtshöhe, wobei allerdings Schloss-, Nonnen- und Erzengasse ungewöhnlich abweichen. ${ }^{8}$ Diese Abweichungen können als Indiz für frühere Veränderungen angesehen werden.

\begin{tabular}{|l|l|l|}
\hline Gassen & Biere & Steuerwert \\
\hline Fischergasse & 7,7 & 183 \\
\hline Markt/Stadtgasse & 7,6 & 209 \\
\hline Breitegasse & 7,0 & 185 \\
\hline Leipzigergasse & 6,8 & 182 \\
\hline Bäckergasse & 6,7 & 155 \\
\hline Spitalgasse & 6,0 & 90 \\
\hline Schulzengasse & 5,2 & 91 \\
\hline Schlossgasse & 4,6 & 107 \\
\hline Nonnengasse & 4,3 & 102 \\
\hline Stümpfergasse & 3,7 & 68 \\
\hline Kuhgasse & 3,4 & 54 \\
\hline Erzengasse & 3,3 & 78 \\
\hline
\end{tabular}

Tab. 2: Durchschnittliche Anzabl der Biere nach Gassen 1514 bis 1525 und durchschnittliche Steuerwerte der brauberechtigten Grundstücke 1531 in Schock Groschen.

7 Die Grundstücksgrößen sind aus den Flurkarten des Jahres 2008 abgeleitet, da erst zu diesem Zeitpunkt die vollständige Vermessung der Altstadt abgeschlossen worden ist. Früher abgetrennte Flächen wurden zugerechnet, verunklarte Grundstücke nicht in die Auswertung einbezogen.

8 HStA Weimar, EGA, Reg Pp 302, 1 - 17, Türkensteuerregister Torgau 1531. 
Grundsätzlich ist der Auffassung Blaschkes zu folgen, dass eine Burgsiedlung östlich des Marktes um 1100 und die planmäßig angelegte Bürgerstadt bis 1250 entstanden sind. ${ }^{9}$ Die früh aus selbstständigen Siedlungskernen einerseits und Stadterweiterungsflächen außerhalb der Stadtmauern andererseits entstandenen Vorstädte verfügten über keine Braurechte und können hier außer Betracht bleiben. Auch der Bereich zwischen Markt/Fleischmarkt und Schloss, der im Wesentlichen herrschaftlich von Freihäusern, Freihöfen und geistlichen Gebäuden besetzt war, hatte keine Braurechte, sofern von den durch die Landesherrschaft befristet gewährten Gnadenbieren abgesehen wird.

Eine besondere Situation ist für den Bereich der Schlossgasse und wohl auch der Nonnengasse vorhanden. Bei archäologischen Untersuchungen 1987/89 in der Schlossgasse wurde festgestellt, dass dieser Bereich durch Aufschüttungen aus Brandschutt mit bis zu 15 m Mächtigkeit, der nur aus dem großen Flächenbrand von 1442 herrühren kann, als Stadterweiterungsfläche angesehen werden muss. Damit wäre hier die Vergabe von Braurechten erst nach 1442 anzusetzen und ist die Annahme Blaschkes, dass die alte Fernhandelsstraße durch die Schlossgasse zum Schlosstor geführt hat, in Frage zu stellen. ${ }^{10}$

Ansonsten bestätigt die Braurechtskarte die bisherige Auffassung, dass die planmäßig angelegte Bürgerstadt ausgehend vom Marktbereich mit ihren Hauptgassen im Rahmen eines einheitlichen Gründungsaktes entstanden ist.

\section{Bierproduktion}

Die Tranksteuern wurden in drei Fristen erhoben: von Michaelis (29. September) bis Weihnachten (Jahresende), von Circumcisio domini (1. Januar) bis Ostern und von Ostern bis Michaelis. Das Steuerjahr war somit nicht das Kalenderjahr. Der Braubeginn erfolgte nach Michaelis in der Regel im Oktober nach Aufruf des Rates, danach war die Brautätigkeit bis Ostern abzuschließen. Der Ausschank in den Häusern der Brauerben und der Verkauf erreichten zwischen Ostern und Michaelis ihren Höhepunkt.

\begin{tabular}{|l|l|l|l|}
\hline & $\begin{array}{l}\text { 1. Frist } \\
\text { 29.09.-31.12. }\end{array}$ & $\begin{array}{l}\text { 2. Frist } \\
\text { 01.01. - Ostern }\end{array}$ & $\begin{array}{l}\text { 3. Frist } \\
\text { Ostern - 29.09. }\end{array}$ \\
\hline Braumenge & $22,0 \%$ & $78,0 \%$ & - \\
\hline Verkaufsmengen & $14,1 \%$ & $18,5 \%$ & $67,4 \%$ \\
\hline Schankmengen & $28,9 \%$ & $19,2 \%$ & $51,9 \%$ \\
\hline
\end{tabular}

Tab. 3: Durchschnittliche Braw-, Verkaufs- und Schankmengen im Zeitraum 1514 bis 1525 nach Stenerfristen in Prozent.

9 Karlheinz Blaschke, Deutscher Städteatlas, Lieferung II, Nr. 14, Dortmund 1979.

10 Heinz Joachim Vogt, Archäologische Untersuchungen im Altstadtbereich von Torgau, in: Ausgrabungen und Funde 37 (1992), S. 50. 
Aufgrund der ermittelten Braurechte von 1.467 Bieren und der festgelegten höchstmöglichen Fassanzahl von sieben Fässern pro Gebräude lag eine theoretische Gesamtkapazität von 10.269 Fass vor, die jedoch nur zu 70 bis 82 \% in Anspruch genommen wurde.

Die Brautätigkeit führten die vom Rat vereidigten Braumeister im Auftrag der Brauherren in deren Brauhäusern durch. Obwohl eine vollständige Übersicht über die Anzahl der Brauhäuser während des Betrachtungszeitraums nicht vorliegt, kann aufgrund späterer Überlieferung davon ausgegangen werden, dass es insgesamt etwa 220 Brauhäuser gegeben hat. Brauerben mit sechs und mehr Bieren verfügten grundsätzlich über ein eigenes Brauhaus. Dies galt für Brauerben mit fünf Bieren nur zu etwa $86 \%$, mit vier Bieren zu etwa $75 \%$, mit drei Bieren zu etwa $50 \%$. Brauerben mit ein bis zwei Bieren hatten kein eigenes Brauhaus. Die kleineren Brauerben mussten ihr Braurecht auf Mietbasis wahrnehmen. ${ }^{11}$

Während der Anteil des Ausschankes in der Stadt selbst von 32,8 \% der Produktion 1515/16 auf $18 \%$ im Braujahr 1523/24 fiel, stieg der Anteil des Verkaufs und der Bierspenden in denselben Braujahren von 56,4 \% auf $78 \%$. Der jährliche Restbestand vor Braubeginn sank 1523/24 auf $4 \%$. Neben dem Verdacht, dass der schwer kontrollierbare Ausschank, der allein besteuert wurde, nicht vollständig abgerechnet worden ist, können auch Jahre mit erhöhter Sterblichkeit, die 1516 und 1521 herrschten, Einfluss gehabt haben. ${ }^{12}$ Im Übrigen wird davon auszugehen sein, dass der Fassverkauf an Abnehmer außerhalb der Stadt bei weniger Aufwand mit höheren Erlösen verbunden gewesen ist. Für den Eigenbedarf der Bürger standen ja auch die steuerfreien Nachbiere in größeren Mengen zur Verfügung. Die gestiegenen Verkaufsmengen und der geringe Restbestand 1523/24 sind auch Hinweise auf die steigende Beliebtheit des Torgauer Bieres.

\begin{tabular}{|l|l|l|l|l|l|l|l|}
\hline Braujahr & Braumenge & \multicolumn{2}{|l|}{ Verkauf/Spenden } & \multicolumn{2}{l|}{ Ausschank } & \multicolumn{2}{l|}{ Restbestand } \\
\hline & & Fass & $\%$ & Fass & $\%$ & Fass & $\%$ \\
\hline $1514 / 15$ & 8.051 & $4.4681 / 2$ & 55,5 & $2.410^{1 / 2}$ & 30,0 & 1.162 & 14,5 \\
\hline $1515 / 16$ & $7.1561 / 2$ & 4.690 & 56,4 & 2.732 & 32,8 & $9001 / 2$ & 10,8 \\
\hline $1517 / 18$ & 8.465 & 5.181 & 55,6 & $1.809 \frac{1}{2} 2$ & 19,4 & 2.331 & 25,0 \\
\hline $1523 / 24$ & 8.372 & $6.6001 / 2$ & 78,0 & $1.506 \frac{1}{2} / 2$ & 18,0 & 265 & 4,0 \\
\hline
\end{tabular}

Tab. 4: Torgauer Bierproduktion, Verkauf/Spenden, Ausschank und Restbestand zu Michaelis des Jabres.

11 Für den Betrachtungszeitraum liegen nur Einzelnachweise von Brauhäusern für einige Grundstücke vor. Die hier mitgeteilten Angaben wurden auf der Basis eines Gesamtverzeichnisses in Auswertung der Nachweisung der besteuerten Häuser in der Stadt ermittelt (wie Anm. 6).

12 Michael Böhme, Chronik von Torgau, o. J., S. 181 f. (Handschrift, Universitätsbibliothek Halle, Hist. 244). 


\section{Bierverkauf in Städte und Dörfer}

Im Steuerjahr 1523/24 gingen $78 \%$ der Produktion in den Verkauf innerhalb des Kurfürstentums und außer Landes. Der Anteil der Lieferungen nach Städten und nach Dörfern hatte 1514/15 fast die gleiche Höhe, veränderte sich aber bis 1523/24 zugunsten der zahlungskräftigeren Städte. Jetzt bezogen 61 Städte insgesamt 3.669 $1 / 2$ Fass und 145 Dörfer 2.084 Fass. Auffallend ist die durchgängige Abnahme von kleinen Mengen von einem halben bis fünf Fass. Das spricht für die gute Qualität, aber auch den höheren Preis des Torgauer Biers, das auch als Festtagsbier diente.

\begin{tabular}{|c|c|c|c|}
\hline \multirow{8}{*}{ 1. Städte } & & $1514 / 15$ & $1523 / 24$ \\
\hline & $\begin{array}{l}\text { Anzahl gesamt } \\
\text { davon Abnahme (Fass) }\end{array}$ & 54 & 61 \\
\hline & $1 / 2-5$ & 29 & 28 \\
\hline & $51 / 2-10$ & 6 & 5 \\
\hline & $10 \frac{1}{2}-20$ & 8 & 9 \\
\hline & $201 / 2-30$ & 1 & 6 \\
\hline & $>30$ & 10 & 13 \\
\hline & Gesamtabnahme (Fass) & $1.9741 / 2$ & $3.6691 / 2$ \\
\hline \multirow[t]{7}{*}{ 2. Dörfer } & $\begin{array}{l}\text { Anzahl gesamt } \\
\text { davon Abnahme (Fass) }\end{array}$ & 158 & 145 \\
\hline & $1 / 2-5$ & 73 & 60 \\
\hline & $5 \frac{1}{2}-10$ & 31 & 31 \\
\hline & $101 / 2-20$ & 23 & 20 \\
\hline & $201 / 2-30$ & 12 & 14 \\
\hline & $>30$ & 19 & 20 \\
\hline & Gesamtabnahme (Fass) & 2.019 & 2.084 \\
\hline \multirow[t]{2}{*}{$\begin{array}{l}\text { 3. Städte u. } \\
\text { Dörfer (Fass) }\end{array}$} & & $3.9931 / 2$ & 6.054 \\
\hline & davon Städte \% & 49,4 & 65,6 \\
\hline
\end{tabular}

Tab. 5: Liefermengen in Städte und Dörfer.

Der Hauptbezugsraum war mit etwa $60 \mathrm{~km}$ Umkreis begrenzt. Eine deutliche Konzentration der Abnehmer bestand im Raum Leipzig. Der Leipziger Rat, der über das alleinige Schankrecht für fremde Biere innerhalb der Stadt verfügte, und das Kollegium der Universität Leipzig, das außerhalb des Stadtrechts bezog, standen dabei an der Spitze. Hier erhöhte sich die Bezugsmenge von 1513/14 mit 851 Fass auf 1523/24 mit 1.610 Fass. Nach Leipzig folgte Halle mit einer Steigerung von $2261 / 2$ auf 752 Fass. Delitzsch, Eilenburg, Naunhof und Schkeuditz bezogen zwischen 100 und 300 Fass. Seit 1522 wurden Lieferungen in die Niederlande registriert, sie stiegen bis $1523 / 24$ auf 145 Fass. (Tab. 6 und Abb. 2) 


\begin{tabular}{|l|l|l|}
\hline & $1514 / 15$ & $1523 / 24$ \\
\hline Delitzsch & 83 & 123 \\
\hline Eilenburg & 150 & $2781 / 2$ \\
\hline Halle & $2261 / 2$ & 752 \\
\hline Leipzig & 851 & 1.610 \\
\hline Magdeburg & - & $11 / 2$ \\
\hline Naunhof & $641 / 2$ & 105 \\
\hline Schkeuditz & $781 / 2$ & $1901 / 2$ \\
\hline Taucha & 34 & 88 \\
\hline Wittenberg & 28 & 41 \\
\hline Niederlande & - & 145 \\
\hline
\end{tabular}

Tab. 6: Liefermengen an Städte (Auswabl) und in die Niederlande.

Für Biertransporte nach Leipzig liegt eine Auswertung der Geleitsbücher des Geleits Eilenburg im Zeitraum von Walpurgis (1. Mai) 1524 bis Walpurgis (30. April) 1525 vor. Danach haben in diesem Zeitraum 221 Transporte mit 1.040 Fass Bier und weitere 33 Transporte Studentenbier ohne Angabe der Fassanzahl stattgefunden.

Der Transport nach Leipzig erfolgte grundsätzlich, ausgenommen das Studentenbier für die Universität, durch Leipziger Ratsbierführer. Ob bei den Leertransporten nach Torgau auch die Leerfässer zurückgeliefert wurden, ist nicht ersichtlich. Je Wagen wurden vier bis fünf, höchstens jedoch sechs Fass geladen. Die Transporte erfolgten ganzjährig, in der Regel mit zehn bis zwanzig Wagen monatlich, im August 1524 mit 35 und im Januar 1525 mit 25 Wagen. Studentenbier wurde vorrangig von April bis Juli bezogen. ${ }^{13}$

Von den Dörfern der Umgebung bezogen diejenigen, die dem Bierzwang innerhalb der städtischen Bannmeile unterlagen, die größten Mengen. Käufer waren vorwiegend die Krüger, darüber hinaus in kleinen Mengen auch die Bauern. An der Spitze der Lieferungen stand 1514/15 Audenhain mit 106 1/2 Fass, Zschakau bezog 93 Fass, Zeckritz 83 1⁄2, Klitzschen, Mockrehna, Wahren, Weidenhain und Zschepplin nahmen je 40 bis 50 Fass ab. Kloster Nimbschen bezog zwischen 23 und 29 Fass.

13 Dieter Postier, Der die Elbe überschreitende Warenverkehr [...], Semesterarbeit am pädagogischen Institut Leipzig 1972 [Ms], S. 27 f. 
Jürgen Herzog



3.
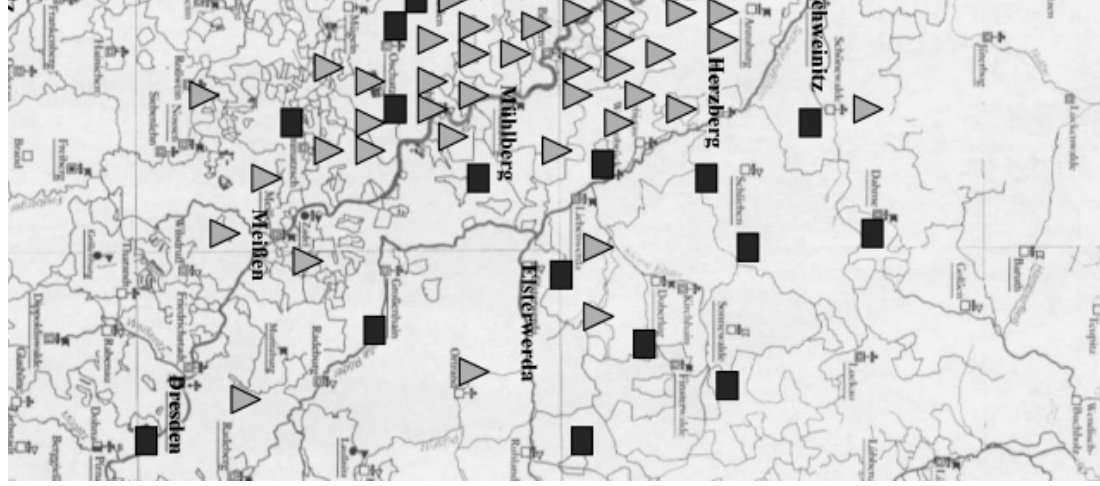

Abb. 2: Bierlieferungen 1514/15 an Städte $\square$ und Dörfer $\triangle$. 


\begin{tabular}{|l|l|l|}
\hline & $1514 / 15$ & $1523 / 24$ \\
\hline Audenhain & $1061 / 2$ & $991 / 2$ \\
\hline Klitzschen & 48 & 25 \\
\hline Mockrehna & 47 & 66 \\
\hline Süptitz & 39 & $121 / 2$ \\
\hline Wahren & 43 & 35 \\
\hline Weidenhain & 57 & 41 \\
\hline Wildenhain & $371 / 2$ & 38 \\
\hline Zeckritz & $831 / 2$ & 26 \\
\hline Zschakau & 93 & 89 \\
\hline Zschepplin & 45 & $371 / 2$ \\
\hline Zschocher & $31 \frac{1}{2} 2$ & $91 \frac{1}{2}$ \\
\hline Kloster Nimbschen & $231 / 2$ & $291 / 2$ \\
\hline
\end{tabular}

Tab. 7: Liefermengen in Dörfer (Auswabl) und Kloster Nimbschen in Fass.

In geringen Mengen wurde das Bier auch über große Strecken transportiert. So fanden z. B. Lieferungen nach Erfurt, Weimar, Köthen, Zerbst und Belzig statt. Die Transporte erfolgten nach dem Abholprinzip. Für einen Bierhandel der Abnehmer gibt es keinerlei Anhaltspunkte. Das schließt nicht aus, dass Bier innerhalb der Städte fassweise verkauft und zuweilen auch als Geschenk weitergegeben wurde.

\section{Bier am kurfürstlichen Hof}

Der kurfürstliche Hof auf Schloss Torgau bezog im Auswertungszeitraum durchschnittlich 144 Fass pro Jahr, darunter 1515/16 308 Fass. Zunehmend erfolgten Lieferungen nach Lochau, bis zu 31 Fass 1523/24, selten nach Wittenberg und Weimar. Schlüsse für den Gesamtbedarf des Hofes lassen sich daraus nicht ableiten, da die Residenzen, so auch Schloss Torgau, eigene Brauhäuser besaßen und auch andere Biere neben dem Torgauer bezogen.

\begin{tabular}{|l|l|l|l|l|l|l|l|l|}
\hline \multicolumn{3}{|l}{$1514 / 15$} & \multicolumn{2}{l}{$1515 / 16$} & $1517 / 18$ & $1523 / 24$ \\
\hline & Anz. & Fass & Anz. & Fass & Anz. & Fass & Anz. & Fass \\
\hline Torgau & 22 & 118 & 42 & 308 & 23 & 71 & 17 & 78 \\
\hline $\begin{array}{l}\text { Wittenberg } \\
\text { Lochau }\end{array}$ & $\jmath 9$ & \multirow{2}{*}{$201 / 2$} & 1 & 1 & -- & -- & -- & -- \\
\hline Weimar & -- & -- & 1 & 2 & -- & -- & -- & -- \\
\hline
\end{tabular}

Tab. 8: Anzabl der Lieferungen und Liefermengen für den kurfürstlichen Hof. 
Die Bezugsmengen spiegeln die wechselnde Hofhaltung Kurfürst Friedrichs des Weisen wider, der sich in seinen letzten Lebensjahren häufig auf Schloss Lochau aufhielt. Lieferanten waren vorwiegend die vermögenden Brauherren und Amtsträger der Stadt und des Landes, so z. B. die Bürgermeister Mattes Moschwitz und Claus Hetzer, der Geleitsmann Wolf Metzsch, der Sekretär des Kurfürsten Johann Feil, der Amtsschösser Leonhard Köppe und der Verwalter des Klosters Nimbschen Johann Eisenmann. Darüber hinaus lieferten Dienstleute des Hofes wie Ott Trummeter, Fritz Schützmeisterin, Cuntz Barbirer, Christoff im Rennstall, Heintz Harnischmeister, der Sekretär Hieronimus Rudelauf und der steuerbefreite Hans Muth, die überwiegend mit ihren Berufsnamen in den Tranksteuerregistern genannt werden. Als Einzellieferungen wurden im Braujahr 1516 für den jungen Herrn von Sachsen, zweifelsohne Johann Friedrich, ein Geschenk des Rates von einem Fass und im gleichen Jahr ein weiteres Fass für Herzog Johann registriert.

Zahlreich waren auch Lieferungen an das Dienstgesinde des Hofes. So 1514/15 auf Voglers Wirtschaft zu Lochau, an die Vogelsteller und Wolfsteller in Rosenfeld, an Wagenknechte und den Wildschützen Behem. Simon Jäger vertrank mit den Jägern eineinhalb Fass im Jägerhaus und der Kurfürst spendete den Bauern in Zschakau ein halbes Fass aus nicht angegebenen Gründen.

\section{Rat der Stadt}

Bierlieferungen an den Ratskeller und an die verpachtete Garküche werden in den Tranksteuerregistern nicht erwähnt. Es bleibt damit offen, in welchem Umfang dort Bier ausgeschenkt wurde. Es ist vermutlich davon auszugehen, dass der Ausschank vorrangig in den Bierstuben der Brauerben erfolgte. Als einzige Schänke mit Ausspannung bezog der unter Amtsjurisdiktion stehende Zschackentaler, vor dem Schloss gelegen, Bier, so 1514/15 30 Fass. 1515 trank der Rat zwei Fass Churbier nach der Ratswahl, ein Fass schenkte er der Pfeffingerin, der Frau des kurfürstlichen Türknechts und Landrentmeisters Degenhart Pfeffinger. Der Stadtschreiber Jobst Schedel schenkte dem Rat 1518 sieben Fass frei und Dienst halber. 1522 hatte der Rat 22 Fass von der Magister Hans Sangerin, der Witwe des Bürgermeisters Caspar Sanger, bezogen, die ihm zur Ausfertigung ihres Testaments weitere vier Fass spendete. Martin Luther erhielt im gleichen Jahr ein Fass als Geschenk. ${ }^{14}$

14 Johann Christian August Bürger, Friedrich Joseph Grulich's Denkwürdigkeiten der altsächsischen kurfürstlichen Residenz Torgau aus der Zeit und zur Geschichte der Reformation nebst Anhängen und Lithographien, Torgau 21855, S. 16. 


\section{Handwerk und Dorfgemeinden}

Die aus den Innungsordnungen des Handwerks bekannte Praxis, zu Weihnachten und zu Pfingsten das gemeine Bier zu trinken, findet sich in den angegebenen Bierlieferungen wieder. 1514 waren die leistungsstarken Handwerkerinnungen der Böttcher, Kürschner, Kramer und Schneider vertreten, aber auch bereits Vereinigungen der Gesellen und Knechte. Auffallend ist der hohe Bierverkauf an die Schuhknechte mit insgesamt sechs Fass, weitere zwei Fass wurden ihnen als Spende gereicht. Die Böttchergesellen mit fünf Fass tranken das Bier zu Weihnachten bei Böttchermeister Zelling in der Breitegasse. Stadtgeschichtlich wichtig ist, dass damit im frühen 16. Jahrhundert neben den Innungen der Meister auch schon Verbindungen der Gesellen bestanden haben.

\begin{tabular}{|l|l|l|l|}
\hline Weihnachtsbier & & ohne Angabe zum Anlass & \\
\hline Büttnerhandwerk & 3 & Fleischer & $1 / 2$ \\
\hline Kürschnerhandwerk & 2 & Schuhknechte & 1 \\
\hline Kramer & 2 & Schuhknechte zur Spende & 2 \\
\hline Schneider & 1 & Gesellen in Loßwig & 6 \\
\hline Schuhknechte & 5 & Gesellen in Audenhain & 3 \\
\hline Büttnergesellen & 5 & Gesellen in Döbern & $21 / 2$ \\
\hline Schneidergesellen & 2 & Gesellen in Mockritz und Welsau je & 1 \\
\hline Gesellen in Loßwig & $1 / 2$ & Gesellen in Klitzschen, Werdau, Zschackau je & $1 / 2$ \\
\hline & & Knechte in Welsau & $11 / 2$ \\
\hline & & Knechte in Mockritz & $1 / 2$ \\
\hline
\end{tabular}

Tab. 9: Lieferung an die Handwerker der Stadt 1514/15 in Fass.

Wer die Gesellen und Knechte waren, die das Bier in den stadtnahen Dörfern tranken, bleibt offen. Zu vermuten ist, dass sie sich hier preisgünstig eingemietet hatten und in der nahen Stadt ihrer Arbeit nachgingen. Auffallend ist, dass im stadtnahen Loßwig $(2,5 \mathrm{~km})$ der höchste Bezug erfolgte. Auch die Dörfer Welsau $(5 \mathrm{~km})$, Werdau $(3 \mathrm{~km})$, Zschakau $(5 \mathrm{~km})$, Döbern $(5,5 \mathrm{~km})$ waren fußläufig leicht $\mathrm{zu}$ erreichen. Schwerer vorstellbar ist das jedoch bei Audenhain $(12 \mathrm{~km})$ und Klitzschen $(8,5 \mathrm{~km})$. Diese Knechte dürften bäuerliche Knechte gewesen sein. Auch die Dorfgemeinschaften pflegten das gemeine Bier zu Weihnachten zu trinken. Dazu wurden die Einnahmen des Dorfgerichts für die Ausrichtung dieses Festbiers verwendet.

Ungewöhnlich für das frühe 16. Jahrhundert ist es, dass in Loßwig die Gärtner als eigene Käufergruppe erscheinen und sich damit von den bevorrechteten Hüfnern als Gemeinschaft absetzen. Loßwig hatte 152918 besessene Mann mit Hufenbesitz und erst acht Gärtner. ${ }^{15}$

15 Karlheinz Blaschke (Hg.), Historisches Ortsverzeichnis von Sachsen. Neuausgabe (Quellen und Materialien zur sächsischen Geschichte und Volkskunde 2), bearb. von Susanne Baudisch und Karlheinz Blaschke, Halbband 1: A-M, Leipzig 2006, S. 446. 


\begin{tabular}{|ll|lc|}
\hline Mockritz & 3 & Zeckritz, Klitzschen, Mockrehna & 1 \\
Neiden, Weihnachten & 2 & Welsau, Zschackau, Melpitz & $1 / 2$ \\
Audenhain & 2 & Gärtner in Loßwig & 1 \\
Loßwig, Weihnachten & 1 & & \\
\hline
\end{tabular}

Tab. 10: Lieferung an Dorfgemeinden 1514/15 in Fass.

\section{Einzellieferungen und besondere Anlässe}

Die gute Qualität und Beliebtheit des Torgauer Biers kommt in den zahlreichen Einzellieferungen für Festlichkeiten, so für Erstmessen, Verlobungen, Wirtschaften (Hochzeiten) und anderen Gelegenheiten, die Bauern der Dörfer, Bürger und Dienstleute des Hofs bezogen, zum Ausdruck. Auch an die Geistlichkeit wurde Torgauer Bier geliefert, so im Steuerjahr 1514/15 an den Abt von Doberlug acht Fass, das Bistum Wurzen zwölf Fass und das Kloster in Dahme, die Pfarrherrn zu Treben, Schildau, Torgau, Kreischau, Zwethau, Audenhain und den Probst zu Schlieben ein halbes bis vier Fass.

Als Vertreter des Adels und unter ihnen auch kurfürstliche Amtsträger kauften Günther von Holdau zu Rosenfeld, Rat Heinrich Löser zu Pretzsch, Hofmarschall Sebastian von Mistelbach auf Klitzschen, Amtmann Bernhard von Hirschfeld, Auerswald zu Weidenhain, der junge Herr von Lüneburg in Wittenberg, Graf Anhalt in Leipzig, der Pfalzgraf in Wittenberg und der Bischof von Würzburg. Landrentmeister Pfeffinger wurde in seinem Torgauer Haus beliefert. Die Witwe Hans von Holdaus, die Holdyn, schenkte zweieinhalb Fass aus, die Edelleute und der Graf von Anhalt vertranken. Als 1515 der Sekretär und Vertraute von Kurfürst Friedrich dem Weisen, Hans Feil, seine Wirtschaft beging und Margarete, eine Tochter des verstorbenen Bürgermeisters Nickel von Dommitzsch, heiratete, bezog er je zwei Fass von seiner Schwiegermutter, der Dommitzschin, und von Hans Muschwitz für eine sicherlich sehr große Hochzeit. Der Torgauer Apotheker Johann Sehofer schenkte Doctor Pistore in Leipzig, es handelt sich wohl um den Arzt Dr. Simon Pistoris, ein halbes Fass, Hans Sachse eineinhalb Fass dem Mediziner und Arzt Herzog Heinrichs, Dr. Blasius Grünewald. 1518/19 erscheinen auch eine Glashütte und der Torgauer Schützenhof unter den Abnehmern.

Das Bezugsverhalten scheint sich auch in den Folgejahren nicht verändert zu haben. Nach Degenhart Pfeffingers Tod 1519 ließ sich 1524/25 Landrentmeister Hans von Taubenheim, der wie Pfeffinger in der Rittergasse wohnte, beliefern. Im gleichen Jahr auch Dr. Simon Pistoris, der herzogliche Kanzler in Dresden. In den Häusern wurde Ehrbier mit Gästen getrunken, so z. B. 1515 beim Amtsschösser Leonhard Köppe sechseinhalb Fass und bei Michael Schlesinger, einem Fleischermeister, zwei Fass Bier. Hans von Dommitzsch trank 1522 ein Fass mit dem Adel zur Fastnacht und ein Fass mit dem Meißner Bischof Johann von Schleinitz, der am 4. April auf seiner gegen die reformatorische Bewegung gerichteten Visitationsreise in Torgau eintraf. ${ }^{16}$ Ein weiteres Fass Bier kaufte der Bischof von Cuntz 
Pasch. In beträchtlichem Umfang wurde für die Kapelle zum beiligen Kreuz, die 1493 von Kurfürst Friedrich dem Weisen vor seiner Kreuzfahrt ins heilige Land gestiftet worden war, Bier käuflich bezogen. ${ }^{17}$ Diese Kapelle als Wallfahrtsort mit dem Grab Christi hat jährlich bis zu 16 Fass Bier abgenommen, wird also mit einem Ausschank und vielleicht auch einer Herberge für Pilger verbunden gewesen sein. Hier erfolgte die letzte Lieferung 1524.

\section{Bierspenden}

Häufig waren die Bierspenden durch Gott für das Torgauer Franziskanerkloster, die Terminierer des Augustinerklosters Herzberg und des Paulinerklosters Leipzig sowie Spenden ohne weitere Bezugsangaben. Seltener waren Spenden für das Kloster Sitzenroda, für Seelenmessen, Seelenbäder und die Schuhknechtsbrüderschaft. Besonders hoch war die Anzahl der Bierspender in den Jahren 1515 bis 1518 mit jährlich 128 Brauherrschaften und 90 Fass Bier, vielleicht auch im Zusammenhang mit der Pestzeit.

\begin{tabular}{|c|c|c|c|c|c|c|c|c|}
\hline & \multicolumn{2}{|c|}{$1514 / 15$} & \multicolumn{2}{|c|}{$1515 / 16$} & \multicolumn{2}{|c|}{$1517 / 18$} & \multicolumn{2}{|c|}{$1523 / 24$} \\
\hline & Anz. & Fass & Anz. & Fass & Anz. & Fass & Anz. & Fass \\
\hline \multicolumn{9}{|l|}{ Klöster durch Gott } \\
\hline Torgau & 63 & 35 & 78 & 45 & 69 & 35 & 5 & 2 \\
\hline Herzberg & 19 & 8 & 14 & $71 / 2$ & 14 & 7 & -- & -- \\
\hline Leipzig & 21 & $10^{1 / 2}$ & 16 & 8 & 15 & 7 & -- & -- \\
\hline Sitzenroda & -- & -- & 4 & 2 & 1 & $1 / 2$ & -- & -- \\
\hline $\begin{array}{l}\text { durch Gott ohne } \\
\text { weitere Angaben }\end{array}$ & 3 & 5 & 16 & $26 \frac{1}{1} 2$ & 22 & 35 & -- & -- \\
\hline $\begin{array}{l}\text { Seelmessen } \\
\text { u. Seelbäder }\end{array}$ & 1 & 1 & 1 & $1 / 2$ & 3 & $2 \frac{1}{2}$ & -- & -- \\
\hline $\begin{array}{l}\text { Schuhknechts- } \\
\text { brüder }\end{array}$ & -- & -- & 1 & 1 & 3 & 3 & -- & -- \\
\hline Spenden ges. & 107 & $59 \frac{1}{1 / 2}$ & 128 & $901 / 2$ & 127 & 90 & 5 & 2 \\
\hline Kapelle heilig Kreuz & 5 & 16 & 13 & $15 \frac{1}{2}$ & 7 & $13 \frac{1 / 2}{2}$ & 1 & $1 / 2$ \\
\hline
\end{tabular}

Tab. 11: Bierspenden durch Gott, Spendenanzabl, Spendenböbe und Verkauf an die Kapelle zum beiligen Kreuz.

Beteiligt an den Spenden für die Klöster waren der Adel, der Rat, kurfürstliches Dienstpersonal, Kaufleute und wohlhabende Handwerker. Die Spenden wurden in gleicher Größenordnung bis zum Steuerjahr 1521/22, also Michaelis 1522, gegeben und kamen vor allem dem Torgauer Franziskanerkloster zugute, während nach 1518 die Bettelmönche aus Herzberg und Leipzig wenig und schließlich

17 Ingetraut Rudolphy, Friedrich der Weise, Göttingen 1989, S. 354. 
nicht mehr bedacht wurden. Den Hauptanteil mit bis zu 45 Fass von bis zu 78 Brauherrschaften erhielten die Torgauer Franziskaner. Zahlreiche Brauherren bedachten sowohl das Torgauer Kloster als auch die beiden Termineien mit je einem halben Fass gleichzeitig. Die lückenhaften Tranksteuerlisten von 1519 bis 1523 lassen durch Hochrechnung des Spendenverhaltens in den einzelnen Fristen zu, die Spendenhöhe zu schätzen. Danach erhielt das Torgauer Kloster 1518/19 etwa 32 Fass, 1521/22 etwa 49 Fass, 1522/23 etwa 14 Fass.

Ab 1522 verminderten sich die Zuwendungen abrupt, 1523/24 spendeten nur noch fünf Brauherren zwei Fass an die Franziskaner in Torgau, 1524/25 wurden die Klöster nicht mehr bedacht. Aus diesem Verhalten der Brauherren ist abzuleiten, dass die reformatorische Bewegung ab $1523 \mathrm{zu}$ einem vollständigen Gesinnungswandel geführt hatte. Jetzt traten andere Verhaltensweisen auf. 1523/24 wurde der Pfarrhof (Pfarre, Pfarrherr) mit zweieinhalb Fass beliefert. Der altgläubige Pfarrherr Thomas Moller trank vier Fass, der Amtsschreiber Egidius Pfrund drei Fass mit den Singern. Eym Pfaffen - diese abwertende Benennung trat erstmalig auf - wurde ein halbes Fass geliefert. Diese Veränderungen gingen mit den reformatorischen Ereignissen in der Stadt einher. Bereits 1521 wurden die Messen nicht mehr geachtet, im April 1522 predigte Martin Luther hier, 1523 wurde der radikale Gabriel Zwilling als Prediger durch den Rat berufen. Im selben Jahr verhalf der Torgauer Kaufmann Leonhart Köppe neun Nimbschener Nonnen, unter ihnen Katharina von Bora, zur Flucht aus dem Kloster Nimbschen und 1525 wurde die Auflösung des Franziskanerklosters mit einem Klostersturm eingeleitet. 18

\section{Maßeinheiten und Bierkonsum}

Bier wurde 1514/25 in Fässern, Vierteln und Achteln verkauft. Dabei hatte ein Fass $=$ zwei Viertel $=$ vier Achtel (Tonnen). Die Umrechnung der gebräuchlichen Fassmaße des 16. Jahrhunderts in heutige Litermaße bereitet Schwierigkeiten. In der bisherigen Literatur vorliegende Angaben sind widersprüchlich. Im Kurfürstentum Sachsen wurde 1514 und 1533 für die Biersteuererhebung das wohl einheitliche Maß des Eimers vorgeschrieben. Von jedem Eimer war ein Silbergroschen Steuer zu zahlen. ${ }^{19}$ In Torgau zahlte man fünf Groschen je Fass, das Fass enthielt damit fünf Eimer. Vergleichsweise hielt ein Dresdner Fass 1702 fünfeinhalb Eimer und 25 Kannen oder sieben Schock Kannen mit 0,937 Liter je Kanne. Damit enthielt ein Fass Dresdner Bier rund 394 Liter und ein Fass Torgauer Bier gerundet 335 Liter. Da nach den Torgauer Tranksteuerlisten ein Eimer 66 Kannen fasste, ergeben sich für die Torgauer Kanne 1,0 Liter Inhalt. 1593 wurde ein Fass

18 Jürgen Herzog, Die Stadt Torgau am Vorabend zur bevorzugten kurfürstlichen Residenz (1531), in: Sächsische Heimatblätter 55 (2009), S. 336-354, hier S. 341; und ERICH SCHILD, Luther in Torgau, Halle 1889, S. 6.

19 Burkhard, Ernestinische Landtagsakten (wie Anm. 4), S. 106. 
Torgauer Bier im Leipziger Burgkeller geeicht und enthielt fünf Schock Leipziger Schenkmaßkanne. Bei einem Leipziger Schenkmaß von 1,1 Litern sind das ebenfalls 335 Liter Fassinhalt. ${ }^{20}$ Eine lange Kontinuität der Fassmaße voraussetzend, ist dieser ermittelte Fassinhalt nicht unglaubwürdig.

Wenn 1525 der Kurfürst auf ein Ansuchen des Rates von Torgau mit der Bitte um Minderung der Belastungen antwortete, er wolle die Notwendigkeit nicht glauben, so den teglich und nechtlich mit saufen, schreyen, spielen alles ufs tewers zugebracht würde, erscheint es angebracht, den Pro-Kopf-Verbrauch an Bier zu ermitteln. ${ }^{21}$

Auf der Basis des durchschnittlichen jährlichen Bierverbrauchs ergeben sich pro Kopf in der Stadt Torgau 1514/25 197 Liter. ${ }^{22}$ Für das Jahr 2005 lag der ProKopf-Verbrauch in Deutschland bei 115 Litern. Unter Berücksichtigung anderen Verbraucherverhaltens und der Bedeutung des Biers im 16. Jahrhundert auch als Nahrungsmittel erscheinen diese Angaben glaubhaft.

\section{Bierpreise und Bierumsatz}

Die Tranksteuer betrug fünf Groschen je Fass, selten werden abweichend zweieinhalb Groschen angegeben. Danach wurde das Fass mit 50 Groschen = zwei Gulden acht Groschen bewertet. Der Rat zahlte z. B. 1522 für ein Fass Bier einschließlich Fuhrlohn nach Wittenberg als Geschenk für Martin Luther ein Schock neun Groschen. Bei einem durchaus möglichen Fuhrlohn von 19 Groschen waren das ebenfalls 50 Groschen Fasspreis. ${ }^{23}$ Auf dieser Grundlage errechnet sich für den Zeitraum von 1514 bis 1524 ein Jahresumsatz der Brauherren von gerundet zwischen 17.000 und 20.000 Gulden. Die Einnahmen des städtischen Haushalts betrugen 1523 vergleichsweise 2.800 Gulden. ${ }^{24}$ Damit wird die eingangs zitierte Behauptung, dass das Bier als ,Brot der Stadt` anzusehen ist, glaubhaft.

20 Verwendet wurden für die Ermittlung neben den Tranksteuerlisten FrITZ VerdenHalven, Alte Maß- und Währungssysteme aus dem deutschen Sprachgebiet, Neustadt an der Aisch 1998, S. 27 und S. 51. Hier wird für das Weinmaß die Leipziger Schenkkanne mit 1,204 Liter angegeben. Stadtarchiv Torgau, H 577, Ratsstatuten 1557, fol. 58v. Eichung eines Torgauer Fasses im Leipziger Burgkeller und Einfügung nach fol. 77r, kurfürstliche Anweisung zur Verwendung der Dresdner Fassmaße 1702. HeRberT Pilz, Bier und Bierausschank im alten Leipzig, in: Leipziger Kalender 1996, S. 98-113, hier S. 100. Hier werden ca. 1,3 Liter für die Leipziger Kanne angegeben.

21 Stadtarchiv Torgau, Nr. H 670, Ratsprotokolle 1525, fol. 5 f.

22 Für die Stadt Torgau wurde die Einwohnerzahl von 3.600 angenommen. Grundlage dafür sind das Schossregister der Stadt von 1505 und das Steuerregister 1531. Dazu auch Herzog, Die Stadt Torgau (wie Anm. 18), S. 352; und Ders., Das Schoßregister der Stadt Torgau des Jahres 1505, in: Sächsische Heimatblätter 38 (1992), S. 287-294, hier S. 293.

23 Bürger, Denkwürdigkeiten (wie Anm. 14), S. 16.

24 HStA Weimar, EGA, Reg Bb 3058, Stadtrechnung Torgau 1523. 



is is





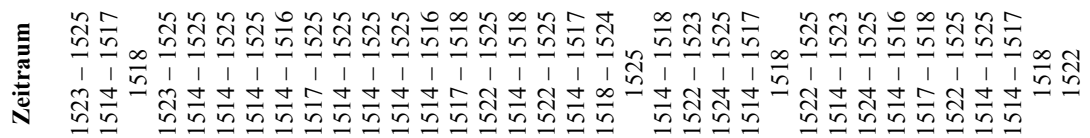



令

$\forall$

$\pi$ 0
8
5
2
2

อิ

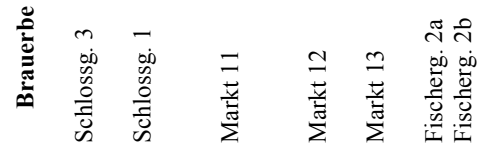

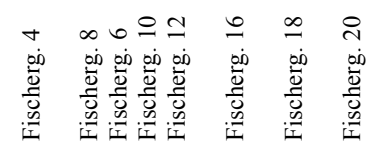

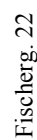

莡

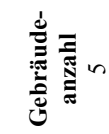

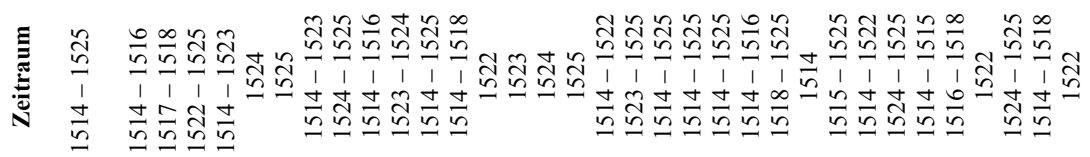

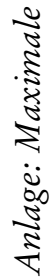

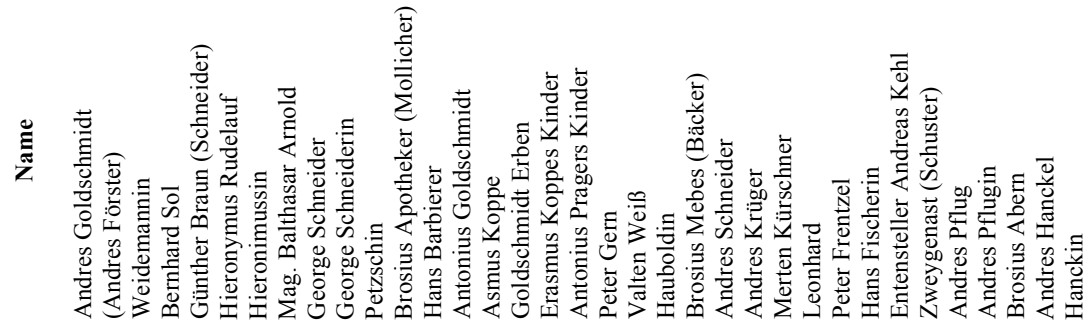


离

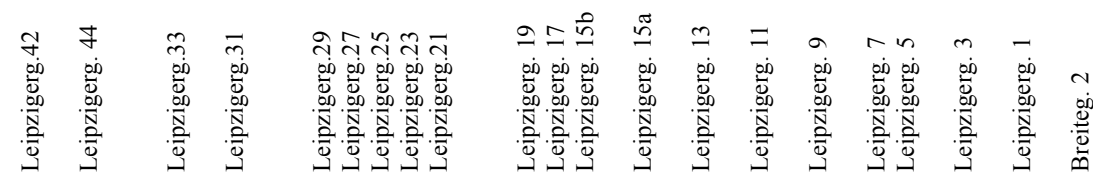

选

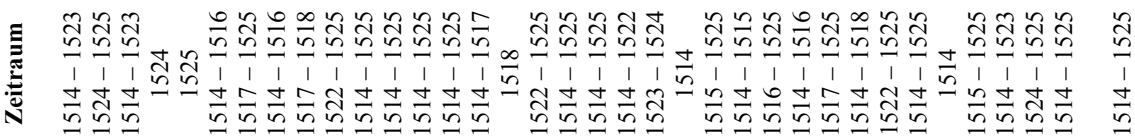

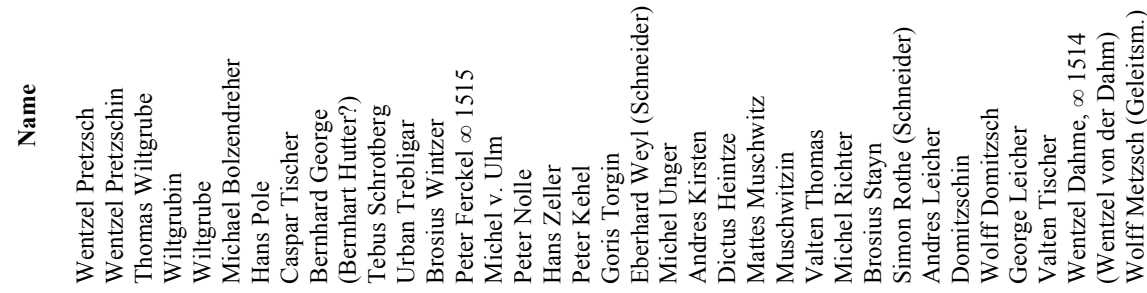

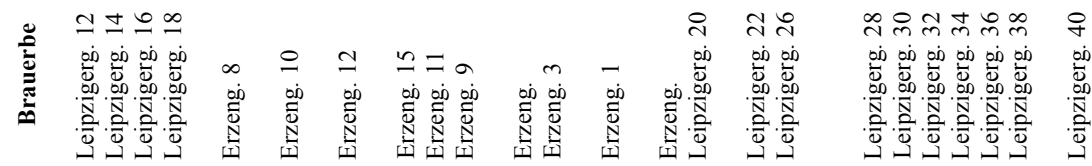

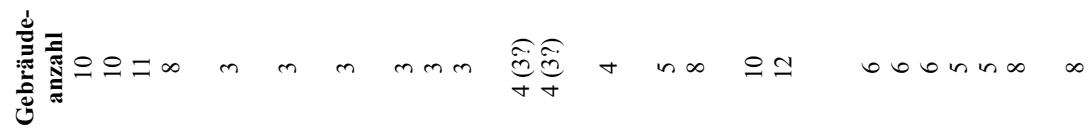

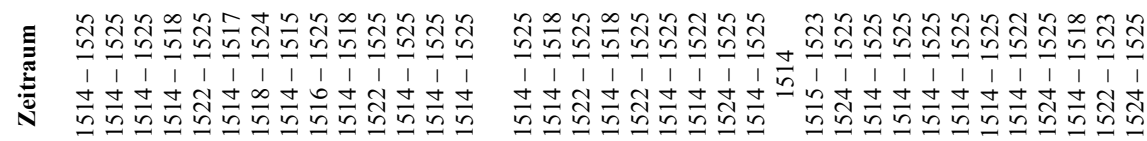

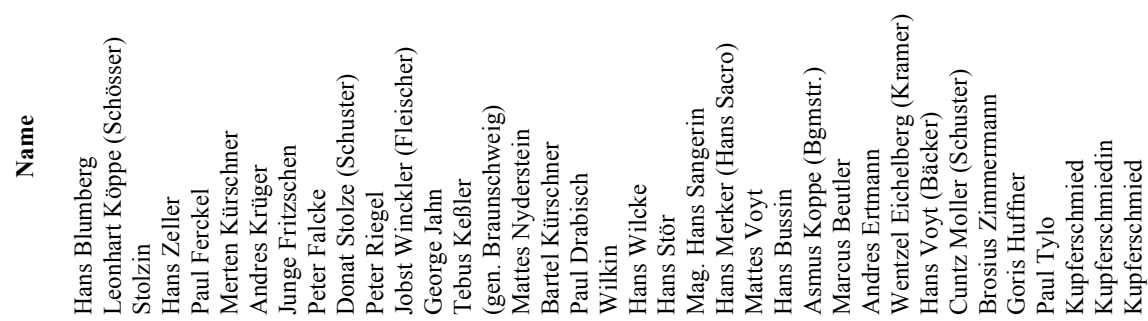




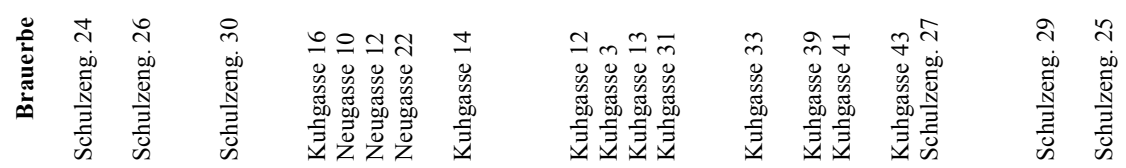

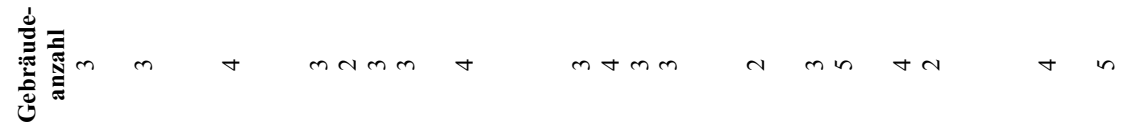

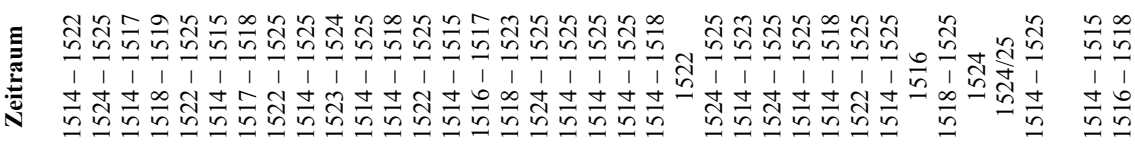

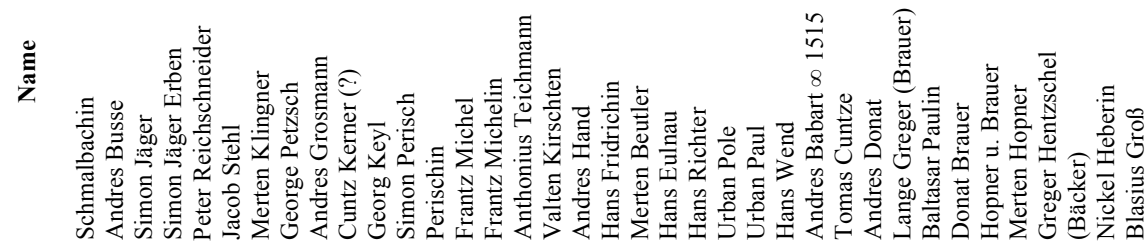

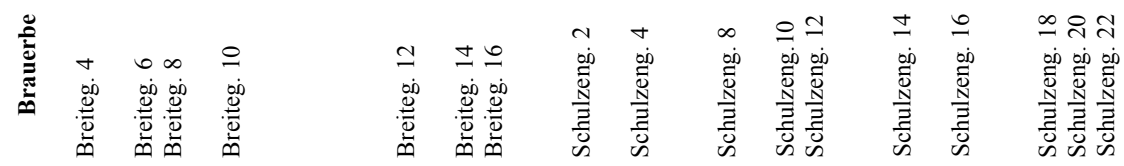

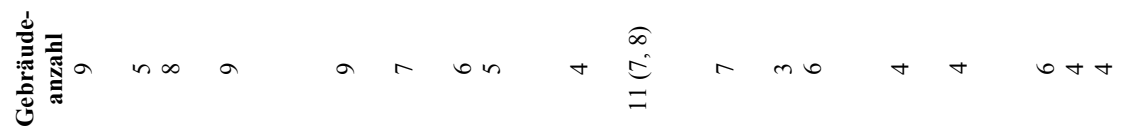

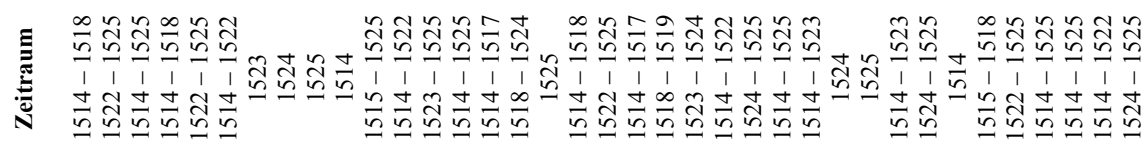

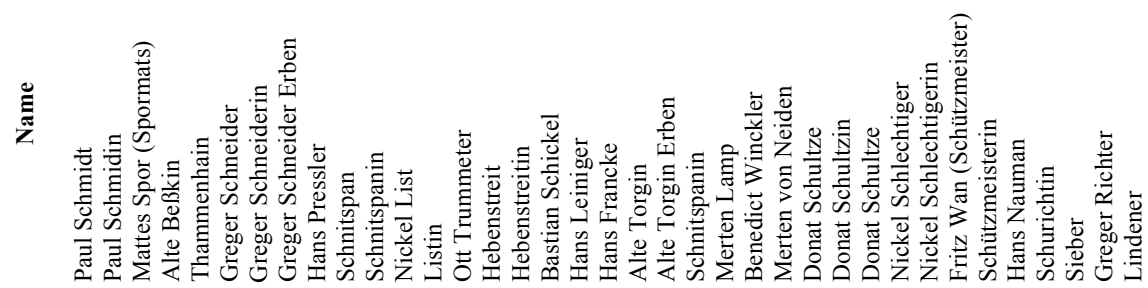




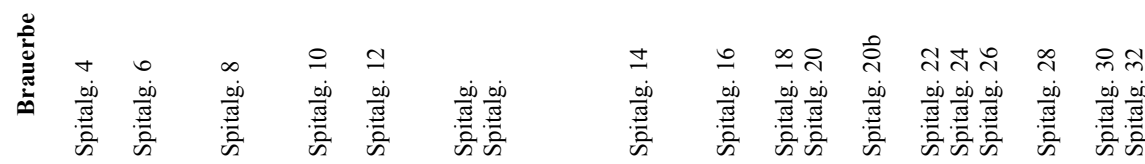

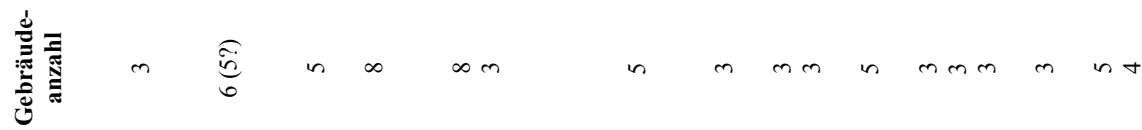



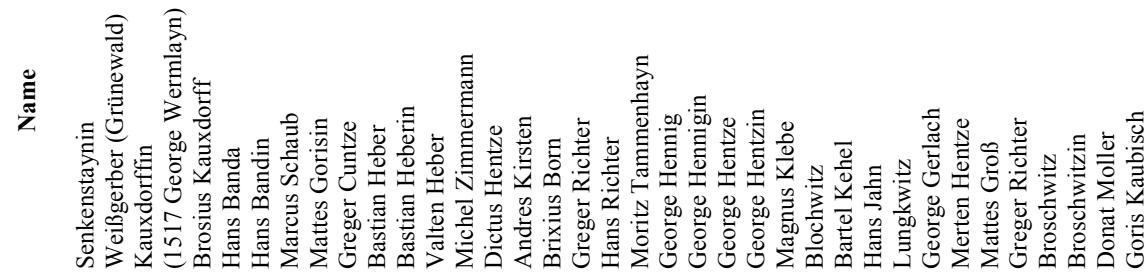

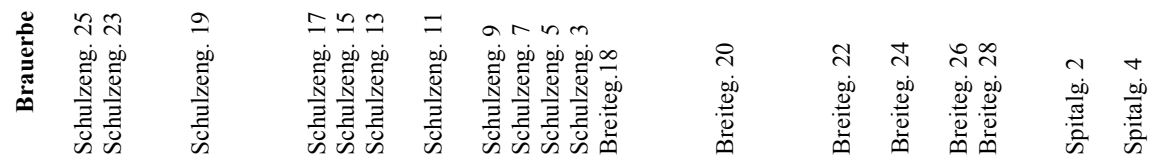
苞

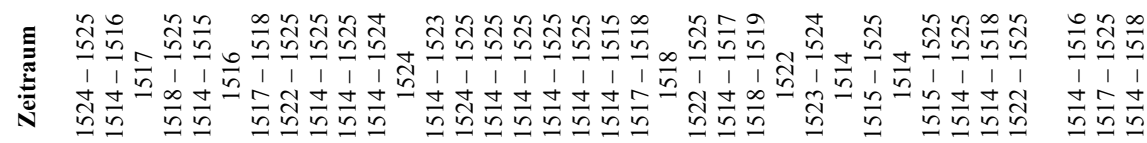

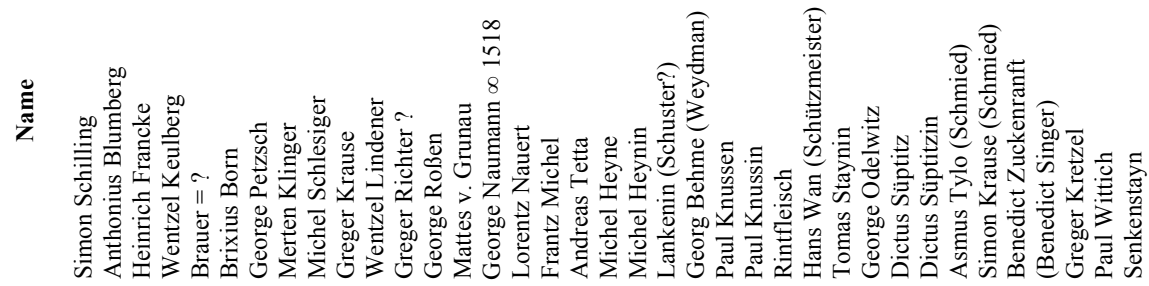




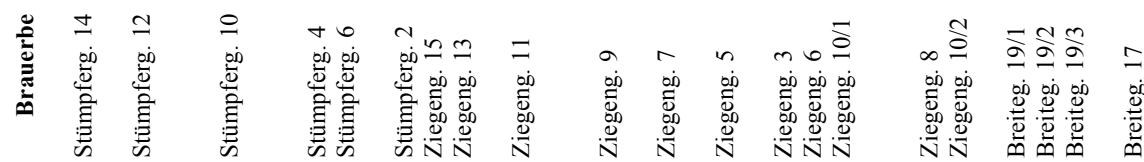

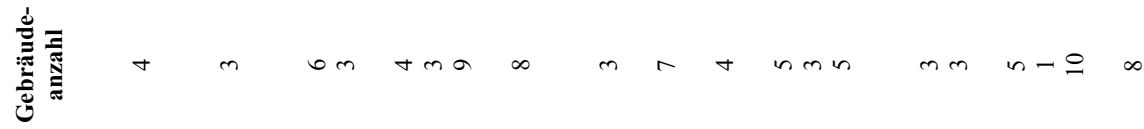

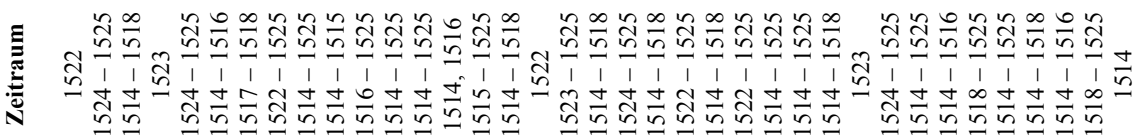



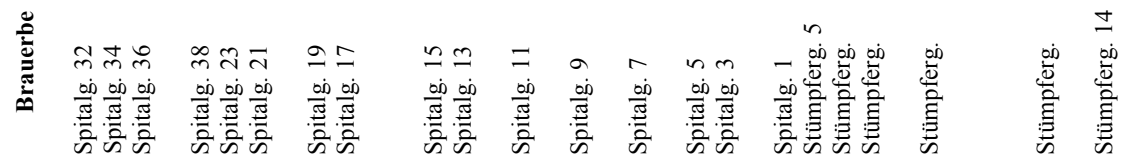

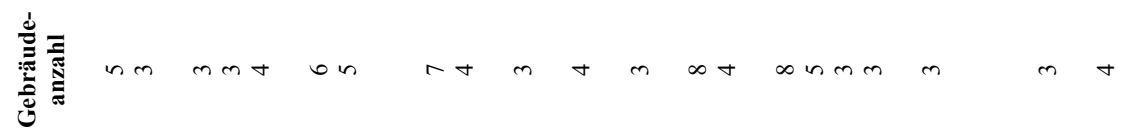

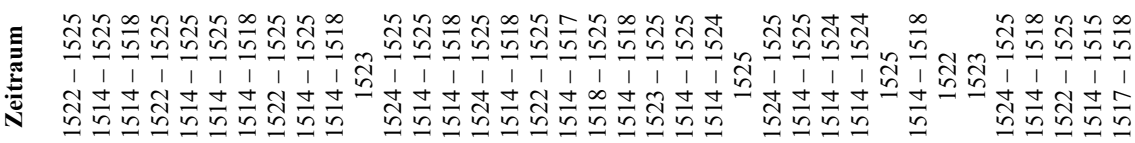

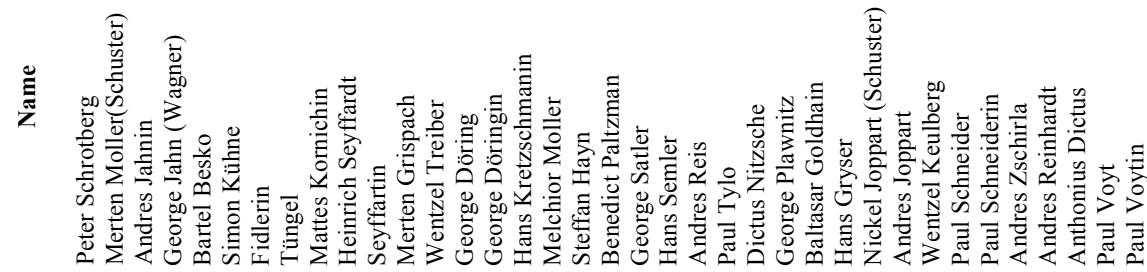




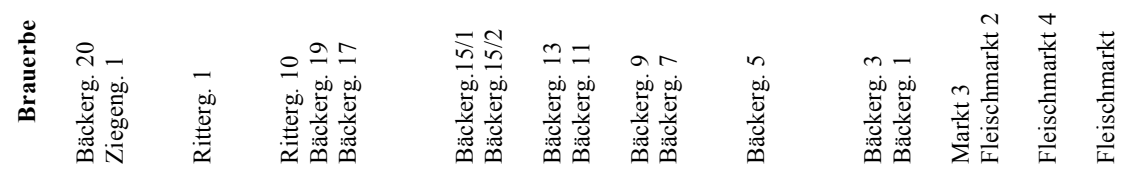

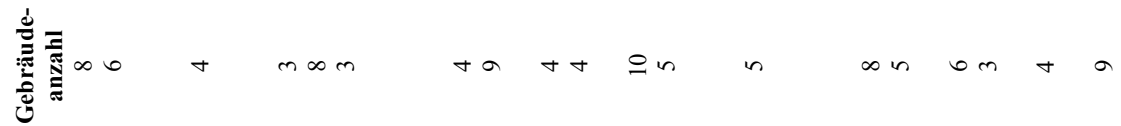

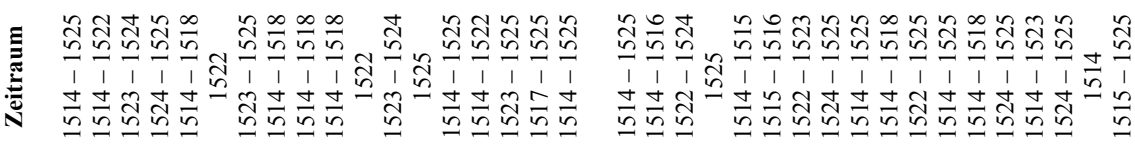

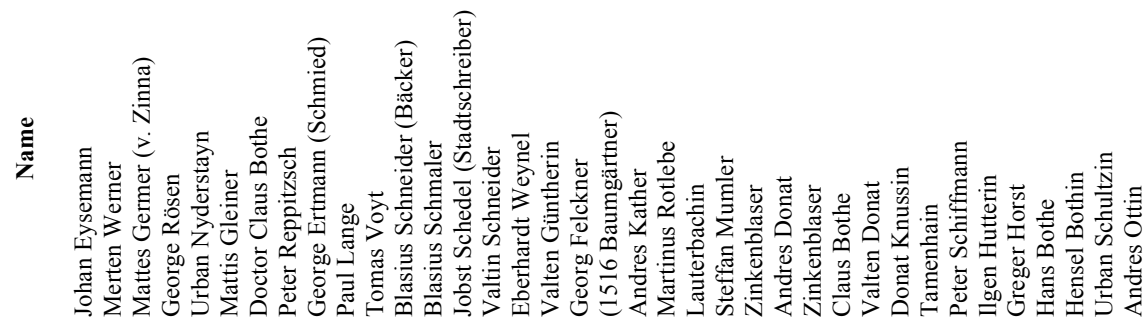

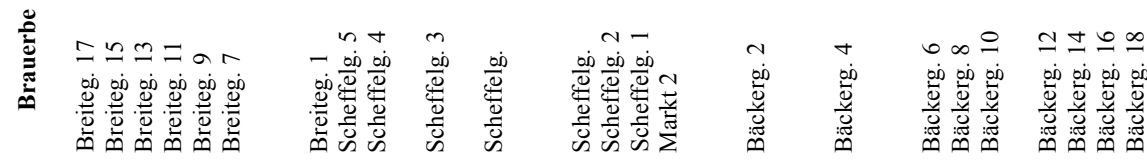



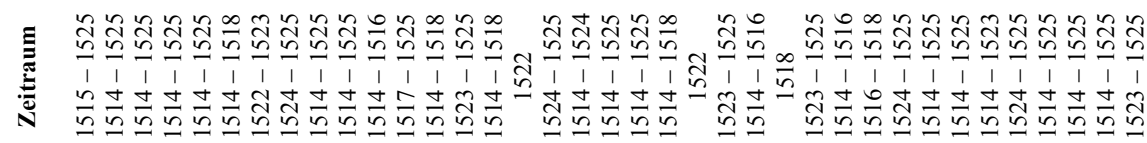








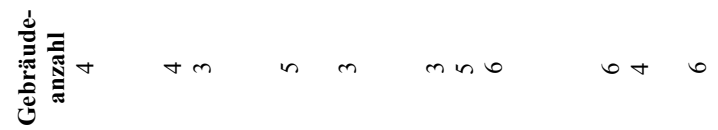

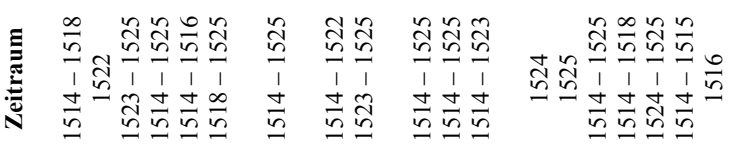

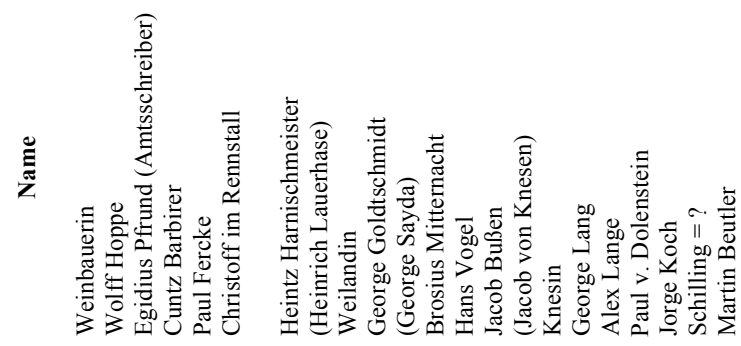

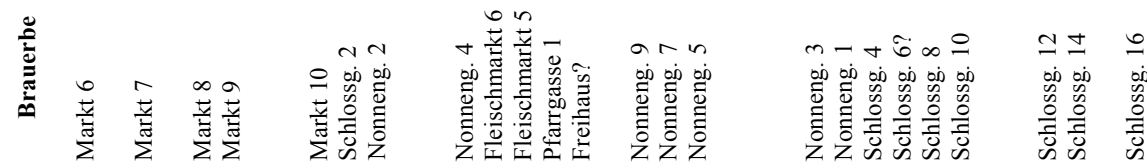

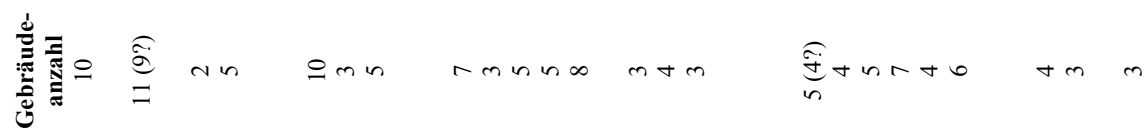



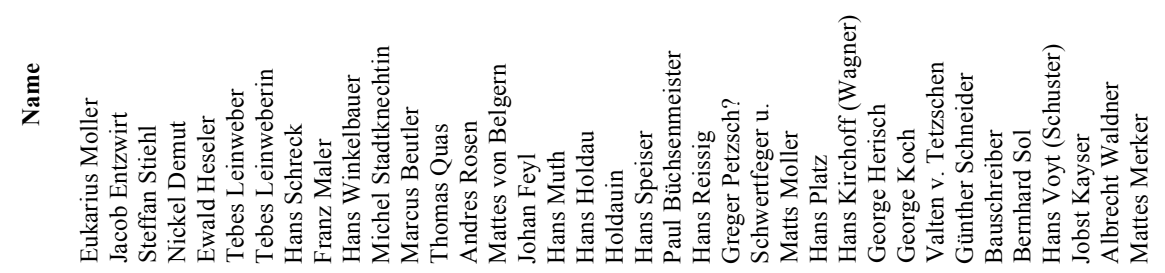

